\title{
Cardiac Autonomic Nervous System Activity during Slow Breathing in Supine Position
}

\author{
Satoru Kai (D), Koji Nagino, Takuma Aoki, Tatsuya Imura, Keita Kiyoshima, \\ Yoshinobu Satake, Kaname Matsuura, Kota Mima, Shoki Yasuoka, and Akinobu Yabuuchi
}

\author{
Division of Physical Therapy, Department of Rehabilitation Sciences, Faculty of Allied Health Sciences, Kansai University of \\ Welfare Sciences, 3-11-1 Asahigaoka, Kashiwara City, Osaka 582-0026, Japan \\ Correspondence should be addressed to Satoru Kai; misakinosora@gmail.com
}

Received 22 November 2020; Revised 20 February 2021; Accepted 23 February 2021; Published 27 February 2021

Academic Editor: Francesco Giallauria

Copyright (c) 2021 Satoru Kai et al. This is an open access article distributed under the Creative Commons Attribution License, which permits unrestricted use, distribution, and reproduction in any medium, provided the original work is properly cited.

\begin{abstract}
The purpose of this study is to clarify cardiac autonomic nervous system activity during slow breathing exercises in a supine position. Eighteen healthy young males were participated. Heart rate variability was measured for 5 minutes at rest, 5 minutes at slow breathing, and then 5 minutes at rest. As a result, the LF/HF ratio increased with slow breathing, but HF value did not change. We suggest that the increased LF/HF ratio may be due to increased airway resistance. Cardiac autonomic nervous system activity during slow breathing in the supine position was revealed.
\end{abstract}

\section{Introduction}

Heart rate variability (HRV) is a popular research tool for assessing cardiac autonomic nervous system (ANS). HRV has also been used in studies to monitor the recovery of heart rate after exercise [1] and to reveal changes due to different postures [2-7]. However, HRV has not been used in studies that reveal cardiac ANS activity during exercise intervention in a supine position. Therefore, it is unclear how exercise intervention in the supine position affects cardiac ANS activity.

With aging, overall cardiac ANS activity and vagal nerve activity decrease. Therefore, it is desired to develop a method for activating cardiac ANS activity. Previous studies have reported that cardiac ANS activity is enhanced by interval training and slow breathing exercise $[1,8]$. However, disuse disorders occur due to aging; it is necessary to ensure safety and security by appropriate methods. Since physiotherapists often examine patients in the supine position, we will clarify the response of cardiac ANS activity to the subjects' supine position due to slow breathing load. The important thing is to clarify cardiac ANS activity during exercise intervention in the supine position and apply it to the physiotherapy program. Therefore, first, the purpose of this study is to clarify cardiac ANS activity during slow breathing in the supine position.

\section{Materials and Methods}

Eighteen healthy young males (age $20 \pm 1$ years (mean \pm S.D.), height $171 \pm 5 \mathrm{~cm}$, body mass $68 \pm 18 \mathrm{~kg}$, and BMI $23 \pm 6$ $\mathrm{kg} \cdot \mathrm{m}^{-2}$ ) were included. To clarify the effect of slow breathing in the supine position, rest was performed in the supine position for 5 minutes, slow breathing in the supine position for 5 minutes, and then resting in the supine position for 5 minutes. Slow breathing was performed under the conditions of exhalation at 6 seconds and inspiration at 4 seconds. Diet, smoking, caffeine intake, and alcohol consumption that affect autonomic nervous activity were prohibited from 2 hours before the measurement. The measurement was carried out in an environment where the room temperature was 24.0 to $26.6^{\circ} \mathrm{C}$ and the humidity was 50.8 to $61.8 \%$. To investigate cardiac ANS activity, an electrocardiogram electrode was attached to the chest, and the data were wirelessly sent to a PC for analysis. The electrocardiogram was monitored by binary light recorder (GMS Company) during the experiment for HRV analysis. Power spectra obtained from spectral analysis were defined as two components: $0.04 \sim 0.15 \mathrm{~Hz}$ (low 
frequency (LF)) and 0.15 0.4 Hz (high frequency (HF)). HF power almost entirely mediated by vagal nerve activity [9] and was affected by the vagal nerve activity generated by respiration $[10,11]$. LF power reflects the mixed modulation of vagal and sympathetic nerve activities [12]. The ratio of LF power to HF power (LF/HF) was considered to reflect the sympathovagal balance, and high values suggested a sympathetic predominance [13]. Therefore, according to previous studies, HF power was used as an index of vagal nerve activity, and LF/HF ratio was used as an index of sympathovagal balance. HF power and LF/HF ratio were logarithmically transformed and shown as $\ln \mathrm{HF}$ and $\ln \mathrm{LF} / \mathrm{HF}$. LF power and LF + HF values, which indicate other autonomic nervous activities, were also used as indicators, and the logarithmically transformed $\ln \mathrm{LF}$ and $\ln \mathrm{LF}+\mathrm{HF}$ values were also used as indicators.

For statistical analysis, SPSS for windows ver. 22.0 (SPSS Inc.) was used, and after the normality test, one-way analysis of variance with repeated measures and multiple comparison test were performed. The significance level was $5 \%$.

Informed consent was obtained from all individual participants included in the study. All procedures performed in this study were in accordance with the ethical standards of the Kansai University of Welfare Sciences Human Research Ethics Committee and with the 1964 Helsinki Declaration and its later amendments or comparable ethical standards.

\section{Results}

The results of each value at rest, slow breathing, and subsequent rest in the supine position are presented in Table 1.

The heart rate values at rest, slow breathing, and subsequent rest in the supine position were $75 \pm 10$ at rest, $76 \pm 9$ at slow breathing, and $73 \pm 8$ at subsequent rest. There was no statistically significant difference among the three groups.

\section{Discussion}

Slow breathing in the supine position revealed that sympathovagal balance activity was activated and vagal nerve activity was unchanged. In the previous study [2], the value of the $\ln \mathrm{LF} / \mathrm{HF}$ ratio at rest in the supine position was $0.2 \pm 0.8$ for males and $-0.3 \pm 0.9$ for females. In the results of this study, it is $0.8 \pm 0.9$, which is a little higher, but it can be seen that it is at the same level. In addition, the ln HF value was 3.8 to 6.2 in the previous study [2-7], and $5.5 \pm 1.2$, which is the result of this study, is within that range. The values during slow breathing compared with the values at rest increased to 2.5 \pm 0.5 in the $\ln$ LF/HF ratio, and a statistically significant difference was observed. Ln HF value decreased to $5.0 \pm 1.1$, and a statistically significant difference was observed. Slow breathing increased LF, ln LF, LF + HF, and ln LF + HF values, all of which were statistically significant. These values indicate an increase in cardiac ANS activity other than the HF value because the HF value has neither increased nor decreased. These findings indicate that slow breathing in the supine position stimulates sympathovagal balance activity.
TABLE 1: Resting, slow breathing, and subsequent resting values in the supine position.

\begin{tabular}{lccc}
\hline & Rest & Slow breathing & Subsequent rest \\
\hline HF & $449 \pm 381$ & $449 \pm 381$ & $305 \pm 260$ \\
ln HF & $5.5 \pm 1.2^{*}$ & $5.0 \pm 1.1$ & $5.3 \pm 0.9$ \\
LF/HF ratio & $4.3 \pm 3.5^{*}$ & $15.7 \pm 7.8$ & $4.5 \pm 2.7^{*}$ \\
ln LF/HF ratio & $0.8 \pm 0.9^{*}$ & $2.5 \pm 0.5$ & $1.0 \pm 0.7^{*}$ \\
LF & $926 \pm 818^{*}$ & $2607 \pm 1891$ & $1110 \pm 1222^{*}$ \\
ln LF & $6.3 \pm 0.9^{*}$ & $7.5 \pm 0.9$ & $6.3 \pm 1.1^{*}$ \\
LF + HF & $1375 \pm 1066^{*}$ & $2877 \pm 2108$ & $1415 \pm 1462^{*}$ \\
$\ln$ LF + HF & $6.8 \pm 0.9^{*}$ & $7.5 \pm 0.9$ & $6.7 \pm 0.9^{*}$ \\
\hline
\end{tabular}

*Slow breathing vs. $p<0.05$, multiple comparison test.

It has been found that slow breathing excites vagal nerve activity and reduces the heart rate, with vagal nerve activity predominant [14-16]. Slow breathing has a remarkable impact on cardiac control above and beyond vagal modulation [17]. In addition, the vagal nerve activity is further enhanced by lengthening the exhalation time rather than the inspiration time during slow breathing $[15,16]$. Decreasing the breathing rate increases the magnitude of heart rate variability and respiratory sinus arrhythmia [18]. Increases regularity of heart rate variability suggesting a decreased complexity of cardiac control [19]. Slow breathing suppresses adrenal medulla norepinephrine secretion and sympathetic nerve excitement. Nevertheless, slow breathing in the supine position does not stimulate vagal nerve activity, but stimulates sympathovagal balance activity. However, the load is low, and in the previous study, the ln LF/HF ratio in the resting position was around 2 [1], and the $\ln L F$ value in the resting supine position was in the range of 3.7 to 7.2 [2-7]. The sitting $\ln \mathrm{LF}$ value is in the range 5.2-7.7 [5, 20, 21]. In the results of this study, the $\ln \mathrm{LF} / \mathrm{HF}$ ratio and $\ln \mathrm{LF}$ value during slow breathing are 2.5 and 7.5 , which are close to the values compared with sitting at rest in previous studies. From these results, it is considered that the load on sympathovagal balance is not high. Therefore, slow breathing in the supine position is likely to be a therapeutic exercise that can also be used for elderly people.

Controlled breathing maneuver is used to assess cardiovascular autonomic control [22-27]. Previous studies have verified that decreased breathing rate is responsible for the increased cardiac vagal regulation [28-33]. Results associating breathing control maneuvers with lower breathing rates and the improvement of vagal modulation are still contradictory [33]. A controlled breathing protocol is used as a method of inducing vagal nerve activation. However, an increase in cardiac sympathetic component in healthy subjects at slow breathing $(0.1 \mathrm{~Hz})$ and an improvement in vagal component at faster breathing pattern $(0.2 \mathrm{~Hz})$ have been observed [18, 34]. The results of these previous studies support the results of this study.

As an immediate response, cardiac ANS activity is unlikely to occur even if slow breathing is performed in a stressed state in a sitting position [8]. Continued slow 
breathing training improves autonomic function [14-16]. The results of those previous studies are divided. Slow breathing increased vagal nerve activity and decreased sympathovagal balance activity $[15,16]$, but no change in sympathetic nerve activity [14]. Resting blood pressure changes with slow breathing for 4 weeks [36]. Slow breathing in 55 healthy subjects reduces both systolic and diastolic blood pressures [37].

When the body position changes, various physiological changes occur in the body. When changing from an upright position to a recumbent position, total lung capacity, vital capacity, and residual capacity are generally slightly reduced, but the expiratory reserve is significantly reduced and a compensatory increase in inspiratory reserve is inadequate [38]. Pulmonary mixing is inferior in the supine position than in the upright position $[39,40]$. Mechanical resistance during inspiration is lower at slow respiratory rates and higher in the supine position than in the sitting position [41]. We suggest that mechanical resistance during expiration is higher at slow respiratory rates than at fast respiratory rates, and at slow respiratory rates in the supine position is higher than in the sitting position. In slow respiratory rates in the supine position, during expiration has a higher mechanical resistance than during inspiration. The effects of such airway resistance may also have stimulated the sympathetic nerve activity. The lung is difficult to expand in the supine position. That may also have affected airway resistance. Details will be clarified in the future.

\section{Conclusions}

Slow breathing in the supine position of young healthy males was found to stimulate cardiac ANS activity without HF power. Especially that, the LF/HF ratio increased. We suggest that the increased LF/HF ratio may be due to increased airway resistance. In the future, we will measure in elderly people with or without disease to clarify the effect of cardiac ANS activity during slow breathing in the supine position.

\section{Data Availability}

There is no data availability.

\section{Conflicts of Interest}

The authors declare that there is no conflict of interest regarding the publication of this paper.

\section{Acknowledgments}

The authors would like to thank the participants who volunteered for this study.

\section{References}

[1] S. Kai, K. Nagino, T. Ito et al., "Effectiveness of Moderate Intensity Interval Training as an Index of Autonomic Nervous Activity," Rehabilitation Research and Practice, vol. 2016, Article ID 6209671, 4 pages, 2016.
[2] R. K. Dishman, Y. Nakamura, M. E. Garcia, R. W. Thompson, A. L. Dunn, and S. N. Blair, "Heart rate variability, trait anxiety, and perceived stress among physically fit men and women," International Journal of Psychophysiology, vol. 37, no. 2, pp. 121-133, 2000.

[3] H. Kobayashi, "Does Paced Breathing Improve the Reproducibility of Heart Rate Variability Measurements?," Journal of Physiological Anthropology, vol. 28, no. 5, pp. 225-230, 2009.

[4] G. Piccirillo, F. Vetta, E. Viola et al., "Heart rate and blood pressure variability in obese normotensive subjects," International Journal of Obesity, vol. 22, no. 8, pp. 741-750, 1998.

[5] O. F. Barak, D. G. Jakovljevic, J. Z. Popadic Gacesa, Z. B. Ovcin, D. A. Brodie, and N. G. Grujic, "Heart rate variability before and after cycle exercise in relation to different body positions," Journal of Sports Science and Medicine, vol. 9, no. 2, pp. 176-182, 2010.

[6] H. Kobayashi, "Inter- and Intra-Individual Variations of Heart Rate Variability in Japanese Males," Journal of Physiological Anthropology, vol. 26, no. 2, pp. 173-177, 2007.

[7] C. Abad, R. Kobal, K. Kitamura et al., "Heart rate variability in elite sprinters-Effects of gender and body position," Clinical Physiology and Functional Imaging, vol. 37, no. 4, pp. 442447, 2017.

[8] S. Kai and M. Koga, "Autonomic nerve responses in a psychological stress task and subsequent slow breathing," Journal of Physical Therapy Science, vol. 24, no. 3, pp. 257-259, 2012.

[9] R. D. Berger, J. P. Saul, and R. J. Cohen, "Transfer function analysis of autonomic regulation. I. Canine atrial rate response," American Journal of Physiology, vol. 256, no. 1, pp. H142-H152, 1989.

[10] B. Pomeranz, R. J. B. Macaulay, M. A. Caudill et al.et al., "Assessment of autonomic function in humans by heart rate spectral analysis," American Journal of Physiology, vol. 248, no. 1, pp. H151-H153, 1985.

[11] S. Akselrod, D. Gordon, F. A. Ubel, D. Shannon, A. Berger, and R. Cohen, "Power spectrum analysis of heart rate fluctuation, a quantitative probe of beat-to-beat cardiovascular control," Science, vol. 213, no. 4504, pp. 220-222, 1981.

[12] L. Bernardi, F. Salvucci, R. Suardi et al., "Evidence for an intrinsic mechanism regulating heart rate variability in the transplanted and the intact heart during submaximal dynamic exercise?," Cardiovascular Research, vol. 24, no. 12, pp. 969981, 1990.

[13] M. Pagani, F. Lombardi, S. Guzzetti et al., "Power spectral analysis of heart rate and arterial pressure variabilities as a marker of sympatho-vagal interaction in man and conscious dog," Circulation Research, vol. 59, no. 2, pp. 178-193, 1986.

[14] E. Tharion, P. Samuel, R. Rajalakshmi, G. Gnanasenthil, and R. K. Subramanian, "Influence of deep breathing exercise on spontaneous respiratory rate and heart rate variability: A randomised controlled trial in healthy subjects," Indian Journal of Physiology and Pharmacology, vol. 56, no. 1, pp. 80-87, 2012.

[15] G. K. Pal and S. Velkumary, "Effect of short-term practice of breathing exercises on autonomic functions in normal human volunteers," Indian Journal of Medical Research, vol. 120, pp. 115-121, 2004.

[16] M. Mourya, A. S. Mahajan, N. P. Singh, and A. K. Jain, "Effect of Slow- and Fast-Breathing Exercises on Autonomic Functions in Patients with Essential Hypertension," The Journal of Alternative and complementary medicine, vol. 15, no. 7, pp. 711-717, 2009. 
[17] A. Porta, R. Maestri, V. Bari et al., "Paced Breathing Increases the Redundancy of Cardiorespiratory Control in Healthy Individuals and Chronic Heart Failure Patients," Entropy, vol. 20, no. 12, p. 949, 2018.

[18] T. E. Brown, L. A. Beightol, J. Koh, and D. L. Eckberg, "Important influence of respiration on human R-R interval power spectra is largely ignored," Journal of Applied Physiology, vol. 75, no. 5, pp. 2310-2317, 1993.

[19] A. Porta, S. Guzzetti, N. Montano et al., "Information domain analysis of cardiovascular variability signals: evaluation of regularity, synchronisation and co-ordination," Medical \& Biological Engineering \& Computing, vol. 38, no. 2, pp. 180-188, 2000.

[20] H. al Haddad, P. B. Laursen, D. Chollet, S. Ahmaidi, and M. Buchheit, "Reliability of Resting and Postexercise Heart Rate Measures," International Journal of Sports Medicine, vol. 32, no. 08, pp. 598-605, 2011.

[21] J. D. Kingsley, V. McMillan, and A. Figueroa, "The Effects of 12 Weeks of Resistance Exercise Training on Disease Severity and Autonomic Modulation at Rest and After Acute Leg Resistance Exercise in Women with Fibromyalgia," Archives of Physical Medicine and Rehabilitation, vol. 91, no. 10, pp. 1551-1557, 2010.

[22] G. D. Pinna, R. Maestri, M. T. La Rovere, E. Gobbi, and F. Fanfulla, "Effect of paced breathing on ventilatory and cardiovascular variability parameters during short-term investigations of autonomic function," American Journal of Physiology-Heart and Circulatory Physiology, vol. 290, no. 1, pp. H424-H433, 2006

[23] J. Adams, P. Julian, M. Hubbard et al., "A randomized controlled trial of a controlled breathing protocol on heart rate variability following myocardial infarction or coronary artery bypass graft surgery," Clinical Rehabilitation, vol. 23, no. 9, pp. 782-789, 2009.

[24] C. Stein, P. Dal Lago, J. B. Ferreira, K. R. Casali, and R. D. M. Plentz, "Transcutaneous electrical nerve stimulation at different frequencies on heart rate variability in healthy subjects," Autonomic Neuroscience, vol. 165, no. 2, pp. 205-208, 2011.

[25] A. Porta, T. Bassani, V. Bari, G. D. Pinna, R. Maestri, and S. Guzzetti, "Accounting for respiration is necessary to reliably infer granger causality from cardiovascular variability series," IEEE Transactions on Biomedical Engineering, vol. 59, no. 3, pp. 832-841, 2012.

[26] J. B. Ferreira, R. D. M. Plentz, C. Stein, K. R. Casali, R. Arena, and P. D. Lago, "Inspiratory muscle training reduces blood pressure and sympathetic activity in hypertensive patients: a randomized controlled trial," International Journal of Cardiology, vol. 166, no. 1, pp. 61-67, 2013.

[27] G. V. Krasnikov, M. Y. Tyurina, A. V. Tankanag, G. M. Piskunova, and N. K. Chemeris, "Analysis of heart rate variability and skin blood flow oscillations under deep controlled breathing," Respiratory Physiology \& Neurobiology, vol. 185, no. 3, pp. 562-570, 2013.

[28] N. Montano, T. G. Ruscone, A. Porta, F. Lombardi, M. Pagani, and A. Malliani, "Power spectrum analysis of heart rate variability to assess the changes in sympathovagal balance during graded orthostatic tilt," Circulation, vol. 90, no. 4, pp. 18261831, 1994.

[29] L. Bernardi, J. Wdowczyk-Szulc, C. Valenti et al., "Effects of controlled breathing, mental activity and mental stress with or without verbalization on heart rate variability," Journals of the American College of Cardiology, vol. 35, no. 6, pp. 1462$1469,2000$.

[30] W. H. Fan, J. H. Ko, M. J. Lee, G. Xu, and G. S. Lee, "Response of nasal airway and heart rate variability to controlled nasal breathing," European Archives of Oto-Rhino-Laryngology, vol. 268, no. 4, pp. 547-553, 2011.

[31] R. Cabiddu, S. Cerutti, G. Viardot, S. Werner, and A. M. Bianchi, "Modulation of the sympatho-vagal balance during sleep: frequency domain study of heart rate variability and respiration," Frontiers in Physiology, vol. 3, 2012.

[32] E. Tobaldini, L. Nobili, S. Strada, K. R. Casali, A. Braghiroli, and N. Montano, "Heart rate variability in normal and pathological sleep," Frontiers in Physiology, vol. 4, 2013.

[33] L. A. de Souza, J. B. Ferreira, A. S. d. O. Schein et al., "Optimization of Vagal Stimulation Protocol Based on Spontaneous Breathing Rate," Frontiers in Physiology, vol. 9, 2018.

[34] M. Reimann, C. Friedrich, J. Gasch, H. Reichmann, H. Rüdiger, and T. Ziemssen, "Trigonometric Regressive Spectral Analysis Reliably Maps Dynamic Changes in Baroreflex Sensitivity and Autonomic Tone: The Effect of Gender and Age," PLoS One, vol. 5, no. 8, article e12187, 2010.

[35] D. E. Anderson, J. D. McNeely, and B. G. Windham, "Regular slow-breathing exercise effects on blood pressure and breathing patterns at rest," Journal of Human Hypertension, vol. 24, no. 12, pp. 807-813, 2010.

[36] Z. Zhang, B. Wang, H. Wu, X. Chai, W. Wang, and C. K. Peng, "Effects of slow and regular breathing exercise on cardiopulmonary coupling and blood pressure," Medical \& Biological Engineering \& Computing, vol. 55, no. 2, pp. 327-341, 2017.

[37] A. Hurtado and W. W. Fray, "Studies of total pulmonary capacity and its sub-divisions. III. Changes with body posture," The Journal of Clinical Investigation, vol. 12, no. 5, pp. 825-832, 1933.

[38] E. Blair and J. B. Hickam, "The effect of change in body position on lung volume and intrapulmonary gas mixing in normal subjects," The Journal of Clinical Investigation, vol. 34, no. 3, pp. 383-389, 1955.

[39] C. J. Martin, F. Cline Jr., and H. Marshall, "Lobar alveolar gas concentrations; effect of body position," The Journal of Clinical Investigation, vol. 32, no. 7, pp. 617-621, 1953.

[40] E. O. Attinger, R. G. Monroe, and M. S. Segal, "The mechanics of breathing in different body positions. I. In normal subjects," The Journal of Clinical Investigation, vol. 35, no. 8, pp. 904911, 1956.

[41] J. Mead, "Statics of respiratory system," in Handbook of Physiology, Respiration I, W. O. Fenn and H. Rath, Eds., p. 398, American Physiological Society, Washington DC, 1964. 